Opinion

\title{
The Clinical use of Guidelines for the Pharmacological Treat- ment of All Diseases is Problematic Because of Little Infor- mation about Adverse Effects
}

\author{
Katsuhiro Toda
}

How to cite this paper: Toda, $\mathrm{K}$. (2022). The Clinical use of Guidelines for the Pharmacological Treatment of All Diseases is Problematic Because of Little Information about Adverse Effects. Universal Journal of Pharmacy and Pharmacology, 1(1), 1-3. Retrieved from

https://www.scipublications.com/journal/in-

dex.php/ujpp/article/view/143

Received: September 12, 2021

Accepted: January 13, 2022

Published: January 14, 2022

Copyright: (c) 2022 by the authors. Submitted for possible open access publication under the terms and conditions of the Creative Commons Attribution (CC BY) license (http://creativecommons.org/licenses /by/4.0/).
Department of Orthopedic Surgery, Hiroshima Clinic. 4th floor Fuji Ground, 2-1, Takara-machi, Naka-ku, Hiroshima, 730-0044, Japan

*Correspondence: goutattack@yahoo.co.jp

\begin{abstract}
In a guideline for the pharmacological treatment, the priority is usually based on the strength of the evidence of efficacy. This is academically correct, but not clinically appropriate. Adverse effects are as important as efficacy in clinical practice. There are guidelines for the pharmacological treatment that take adverse effects into account. However, most adverse effects considered are those obtained in double-blind studies of pharmacological treatment performed to prove efficacy. In the treatment guidelines of various diseases, too little effort is spent on pharmacological adverse effects compared to the effort spent on pharmacological efficacy. I would like to make recommendations in the guidelines for the pharmacological treatment of all diseases. The guidelines for the pharmacological treatment of various diseases should be developed with increased efforts to investigate adverse effects. Some guidelines for the pharmacological treatment use systematic reviews and meta-analyses to examine the efficacy of medicine, and similar methods should be used to examine the adverse effects of medicine. It is easy to prioritize medicines based on evidence of efficacy. However, it is not appropriate to prioritize medicines in clinical practice based solely on evidence of efficacy. Adverse effects should also be considered in priority ranking of medicine in clinical practice. In addition to efficacy and adverse effects, price and the degree of off-label prescribing also affect the priority ranking of medicine in clinical practice.
\end{abstract}

Keywords: Guidelines; Pharmacological Treatment; Adverse Effects; Efficacy

Treatment guidelines of various diseases have been reported worldwide including Japan. Attention should be paid when we use guidelines for the pharmacological treatment in clinical practice. In a guideline for the pharmacological treatment, the priority is usually based on the strength of the evidence of efficacy. This is academically correct, but not clinically appropriate.

As mentioned above, in a guideline for the pharmacological treatment, the priority is usually based on the strength of the evidence of efficacy. Pharmaceutical companies support financially when conducting clinical trials of expensive drugs that benefit them. Because there are many clinical trials for expensive drugs, the evidence for expensive drugs is likely to be higher. One systematic review showed a statistically significant association between industry sponsorship and pro-industry conclusions (pooled MantelHaenszel odds ratio, 3.60; 95\% confidence interval, 2.63-4.91) [1]. Because there are not so many clinical trials for inexpensive but effective drugs, they are unlikely to be included in the guideline. Nortriptyline in neuropathic pain or fibromyalgia is a typical example.

The biggest problem is the adverse effects. As mentioned above, in a guideline for the pharmacological treatment of various diseases, the priority is usually based on the strength of the evidence of efficacy. However, adverse effects are as important as efficacy 
in clinical practice. There are guidelines for the pharmacological treatment that take adverse effects into account. However, most adverse effects considered are those obtained in double-blind studies of pharmacological treatment performed to prove efficacy. This causes some problems. 1: Double-blind studies of pharmacological treatment performed to prove efficacy are often performed with financial support of pharmaceutical companies. Studies funded by pharmaceutical companies are more likely to show efficacy and less likely to show adverse effects [1-4]. For example, meta-analyses including an author who were employees of the manufacturer of the assessed antidepressant were 22-fold less likely to have negative statements about the antidepressant than other meta-analyses [1/54 (2\%) vs. 57/131 (44\%); P < 0.001] [4]. On the other hand, no association of industry funding or author's financial conflicts of interest with study outcome was seen in fibromyalgia drug therapy randomized controlled trials [5]. The relationship between conflict of interest and study results is not the essence of this opinion paper. 2: Adverse effects reported in studies other than the double-blind studies of pharmacological treatment performed to prove efficacy are not considered. 3: The study period for double-blind studies of pharmacological treatment is short, usually less than six months. Naturally, adverse effects of open studies for more than one year are not considered. 4: Adverse effects that are not easily noticed, such as sexual dysfunction, dementia, reduced bone density, are not considered. These adverse effects can be identified only in long-term studies.

In the treatment guidelines of various diseases, too little effort is spent on pharmacological adverse effects compared to the effort spent on pharmacological efficacy. I would like to make recommendations in the guidelines for the pharmacological treatment of all diseases. The guidelines for the pharmacological treatment of various diseases should be developed with increased efforts to investigate adverse effects. Some guidelines for the pharmacological treatment use systematic reviews and meta-analyses to examine the efficacy of medicine, and similar methods should be used to examine the adverse effects of medicine. The Delphi method shows recommendations based on the consensus of a number of persons. It is seemingly reasonable. However, even if persons who do not know enough about adverse effects gather to consensus, it is not appropriate. When we judge the usefulness of medicine by comparing its efficacy and adverse effects, it is not appropriate to judge the usefulness of medicine with the difference in the amount of both information. The term risk-benefit ratio is meaningless if there is insufficient information on adverse effects. Adverse effects are particularly problematic in guidelines for the pharmacological treatment of diseases that require long-term pharmacological treatment. My opinion is stale. However, this opinion has been ignored and problematic guidelines for the pharmacological treatment have been used.

Many people may worry about the enormous effort of investigating the adverse effects. I specialize in pharmacological treatment of pain. For more than 10 years, the efficacy and/or adverse effects of antidepressants, anticonvulsants, acetaminophen, non-steroidal anti-inflammatory drugs, proton pump inhibitors, etc. were thoroughly investigated using Pubmed. Based on it, I have personally made guidelines for the pharmacological treatment of some pains. What I personally can do, can also be done by a scientific organization. Antidepressants are prescribed in depression, anxiety disorder, and neuropathic pain (or fibromyalgia), etc. Even if the purpose is different, adverse effects are presumed to be the same. In the case of antidepressants, it saves time if academic societies involved in the disease collaborate to investigate the adverse effects.

It is easy to prioritize medicines based on evidence of efficacy. However, it is not appropriate to prioritize medicines in clinical practice based solely on evidence of efficacy. Adverse effects should also be considered in priority ranking of medicine in clinical practice. In addition to efficacy and adverse effects, price and the degree of off-label prescribing also affect the priority ranking of medicine in clinical practice. At the individual physician level, in addition to efficacy and adverse effects in the literature, efficacy and adverse effects based on personal experience also affect the priority ranking of medicine. 
According to the package insert (which has the same force as the law in court), driving a car is prohibited in Japan without exception while taking all psychotropics including hypnotics other than serotonin noradrenaline reuptake inhibitors. Almost no one including judges, public prosecutors, and drivers of police cars abide by the rule, however, physicians must explain it to patients in Japan. In Japan, this strange system also affects the priority ranking of medicine.

In addition, I would like to make a suggestion. The guidelines for the pharmacological treatment of various diseases are guidelines for starting pharmacological treatment. Please make a guideline for discontinuation when it is ineffective or remarkably effective.

Funding: No funding.

Conflicts of interest: My daughter is an employee of Nippon Zoki Pharmaceutical Co., Ltd.

Ethics approval: Not applicable.

\section{References}

[1] Bekelman, J.E.; Li, Y.: Gross, C.P. Scope and impact of financial conflicts of interest in biomedical research: a systematic review. J.A.M.A. 2003, 289, 454-465.

[2] Sismondo, S. Pharmaceutical company funding and its consequences: a qualitative systematic review. Contemp. Clin. Trials. 2008, 29, 109-113.

[3] Cosgrove, L.; Shi, L.; Creasey, D.E.; Anaya-McKivergan, M.; Myers, J.A.; Huybrechts, K.F. Antidepressants and Breast and Ovarian Cancer Risk: A Review of the Literature and Researchers' Financial Associations with Industry. PLoS. One. 2011, 6, e18210.

[4] Ebrahim, S.; Bance, S.; Athale, A.; Malachowski, C.; Ioannidis, J.P.A. Meta-analyses with industry involvement are massively published and report no caveats for antidepressants. J. Clin. Epidemiol. 2016, 70, 155-163.

[5] Pang, W.K.; Yeter, K.C.; Torralba, K.D.; Spencer, H.J.: Khan, N.A. Financial conflicts of interest and their association with outcome and quality of fibromyalgia drug therapy randomized controlled trials. Int. J. Rheum. Dis. 2015, 18, 606-615. 